\title{
CircRNA_101951 promotes migration and invasion of colorectal cancer cells by regulating the KIF3A-mediated EMT pathway
}

\author{
YUE-FENG LI ${ }^{1}$, FU-LAI PEI ${ }^{1}$ and MING-ZHENG CAO ${ }^{2}$ \\ Departments of ${ }^{1}$ Oncology and ${ }^{2}$ General Surgery, Linyi Central Hospital, Linyi, Shandong 276400, P.R. China
}

Received October 2, 2019; Accepted February 18, 2020

DOI: $10.3892 /$ etm.2020.8600

\begin{abstract}
Colorectal cancer (CRC) is one of the most lethal tumor types worldwide. Circular RNAs (circRNAs), which are covalent closed loops of RNA, perform vital roles for the proliferation and metastasis of a variety of tumor types. In the present study, the expression, function and molecular mechanisms of action of a novel circRNA, circRNA_101951, were examined in CRC. The expression levels of circRNA_101951 in CRC tissue and cell lines were examined using reverse transcription-quantitative (RT-qPCR). Cell proliferation, the clone formation ability, cell apoptosis, the cell cycle and the cell migratory and invasive abilities were examined using MTT assays, colony formation assays, flow cytometric assays, and cell migration and invasion assays, respectively. The effects of circRNA_101951 on Kinesin II family member 3A (KIF3A) related gene expression were examined using RT-qPCR and western blot assays. The results indicated that circRNA_101951 was increased in CRC tissues and cell lines. The downregulation of circRNA_101951 inhibited cell proliferation and colony formation as well as cell migration and invasion of CRC cell lines. In addition, the downregulation of circRNA_101951 blocked the KIF3A-mediated epithelial-mesenchymal transition (EMT) pathway, which was detected by examining the expression levels of KIF3A and EMT related proteins. In conclusion, the current data revealed that circRNA_101951 may act as a potential biomarker for patients with CRC, and provided a novel insight demonstrating that the suppression of circRNA_101951 may be a potential therapeutic strategy for CRC.
\end{abstract}

Correspondence to: Mr. Fu-Lai Pei, Department of Oncology, Linyi Central Hospital, 17 Jiankang Road, Linyi, Shandong 276400, P.R. China

E-mail: FulaiPei@163.com

Key words: circular RNA_101951, migration, invasion, kinesin II family member $3 \mathrm{~A}$, epithelial-mesenchymal transition, colorectal cancer

\section{Introduction}

Currently, colorectal cancer (CRC) is one of the most lethal tumor types worldwide (1). Although there is progress in the clinical treatment of patients with CRC, the overall survival time has not significantly improved in recent years (2). Migration, metastasis and invasion of CRC cells are likely factors that lead to increased recurrence and mortality in patients $(3,4)$. Therefore, the identification of the potential molecular mechanisms of action behind metastasis in CRC is urgently required.

Circular RNAs (circRNAs), which are covalent closed loops of RNA (5-7), are reported to serve vital roles in the proliferation and metastasis of tumors (8-12). circRNAs have also been identified as potential biomarkers for the early diagnosis of a variety of tumor types. For example, homo sapien (hsa)_circ_0000520, which is a novel circRNA biomarker, is involved in gastric carcinoma (13), comprehensive circRNA profiling has also revealed that hsa_circ_0005075 is involved in the development of hepatocellular carcinoma (14). Additionally, hsa_circ_0013958 has been identified as a circRNA and potential novel biomarker for lung adenocarcinoma (15). In addition, circRNAs have been demonstrated to promote the proliferation and metastasis of tumors by sponging miRNA. For example, circRNA-zinc finger RNA binding inhibited cell proliferation and promoted apoptosis in gastric cancer by sponging miR-130a/miR-107 and modulating PTEN (16). CircRNA-proteasome 26S ATPase subunit 3 suppresses the proliferation and metastasis of gastric cancer by acting as a competitive endogenous RNA through sponging miR-296-5p (17). CircRNA-Filamin-binding LIM protein 1 acts to promote hepatocellular cancer progression by sponging miR-346 (18). The aforementioned studies revealed that circRNAs serve a major role in the tumorigenesis of a variety of tumor types.

In the present study, the expression profile of circRNAs in CRC tissues was examined by analyzing the microarray data from the GEO dataset GSE126094. The results revealed that circRNA_101951 was overexpressed in CRC tissues. circRNA_101951 is a novel circRNA and the biological function and the mechanisms of action of circRNA_101951 are currently undetermined. Therefore, the present study demonstrated that circRNA_101951 promoted migration and invasion of CRC cells by regulating the epithelial-mesenchymal transition (EMT). 


\section{Materials and methods}

Patients and specimens. The 62 pairs of CRC tissues and corresponding adjacent normal tissues (obtained $<3 \mathrm{~cm}$ from tumor tissue) were collected from 62 patients ( 32 males and 30 females; age range, 26-88 years) who had undergone surgery in the Oncology Department of Linyi Central Hospital (Linyi, China) between May 2015 to December 2018. None of the patients received preoperative radiotherapy or chemotherapy and all samples were collected according to the guidelines of the Health Insurance Portability and Accountability Act. Written informed consent was obtained from all patients and the study was approved by the Board and Ethics Committee of Linyi Central Hospital (approval no. 2018120102). To explore the survival of 62 patients with CRC, patients were divided into high and low circRNA_101951 expression groups according to the median expression value (10.75). TNM was conducted as previously described (19).

Cell culture. NCM460, a human colon mucosal epithelial cell line (The Cell Bank of Type Culture Collection of the Chinese Academy of Sciences) and four CRC cell lines (SW620, HCT116, SW480 and HT29) were cultured in DMEM (Invitrogen; Thermo Fisher Scientific, Inc.) supplemented with $10 \%$ FBS (Gibco; Thermo Fisher Scientific, Inc.) at $37^{\circ} \mathrm{C}$ with $5 \% \mathrm{CO}_{2}$ atmosphere.

Bioinformatics analysis. The microarray data of the circRNA profiles in CRC tissues and corresponding adjacent normal tissues were collected from the NCBI GEO Datasets (https://www.ncbi.nlm.nih.gov/geo/query/acc. cgi?acc=GSE126094). After applying a $\log 2$ transformation to the data, GEO2R (https://www.ncbi.nlm.nih.gov/geo/geo2r/) was used to analyze the normalized microarray data (20).

Small interfering (si)RNA transfections. The si-circRNA_101951 and si-negative control (NC) were synthesized by Sangon Biotech Co., Ltd. The sequences of the si-circRNA_101951 and si-NC were as follows: 5'-GUCUAG CUUAGAGUCUAU-3' and 5'-CAGAUUCGUUAGAGUCUA U-3', respectively. Lipofectamine ${ }^{\mathrm{TM}} 2000$ (Invitrogen; Thermo Fisher Scientific, Inc.) were used in transient cell transfection. siRNA ( 80 pmol) and Lipofectamine 2000 reagents $(6 \mu \mathrm{l})$ were diluted using $100 \mu \mathrm{l}$ Opti-MEM medium (Invitrogen; Thermo Fisher Scientific, Inc.) and incubated for $2 \mathrm{~min}$ at room temperature. Diluted RNA and transfection reagents were subsequently mixed and incubated at room temperature for $20 \mathrm{~min}$. The mixture was then added to a 6-well plate that was seeded with $2 \times 10^{5}$ cells. Further experiments were performed $48 \mathrm{~h}$ after transfection.

MTT assay. The CRC cell proliferation was examined using MTT assays (Sigma-Aldrich; Merck KGaA) following the manufacturer's instructions. DMSO was used to dissolve the purple formazan and the optical density value at $490 \mathrm{~nm}$ was measured on a microplate reader.

Colony formation assay. Colony formation assays followed the standard protocols as previously described (21). After 2 weeks of incubation at $37^{\circ} \mathrm{C}$ with $5 \% \mathrm{CO}_{2}$, colonies were immobilized using 4\% paraformaldehyde (Sigma-Aldrich; Merck $\mathrm{KGaA}$ ) at room temperature for $10 \mathrm{~min}$ and stained with $2 \%$ crystal violet (Sigma-Aldrich; Merck KGaA) at room temperature for $5 \mathrm{~min}$. Colonies were subsequently imaged with a camera (Nikon Corporation) and analyzed using IPP Image-Pro Plus 6.0 software (Media Cybernetics, Inc.).

Cell cycle analysis. Cell cycle analysis was performed according to the standard protocols of the CycleTEST ${ }^{\mathrm{TM}}$ PLUS DNA Reagent kit (cat. no. 340242, BD Biosciences) and analysis of the cell cycle were determined using the PI reagent. Cells were counted using the BD Accuri ${ }^{\mathrm{TM}} \mathrm{C} 6$ flow cytometer (BD Biosciences) and analyzed using FlowJo ${ }^{\circledR}$ 10.4.1 software.

Cell apoptosis assay. The cell apoptosis assay was performed according to the standard protocols of the BD Pharmingen ${ }^{\mathrm{TM}}$ FITC Annexin V Apoptosis Detection kit (cat. no. 556547, BD Biosciences). The extent of cell apoptosis was then detected using FITC and PI reagents. Cells were counted with BD Accuri ${ }^{\text {TM }}$ C6 (BD Biosciences) and analyzed using FlowJo ${ }^{\circledR}$ 10.4.1 software.

Cell migration. Cells were suspended in DMEM medium (Invitrogen; Thermo Fisher Scientific, Inc.) without serum, and then $3 \times 10^{5}$ cells were seeded into the upper chamber of a transwell insert ( $8.0 \mu \mathrm{m}$; BD Biosciences; 24 well-plate). DMEM medium supplemented with $10 \%$ FBS was added to the lower chamber to act as a chemoattractant. The cells were cultured for $8 \mathrm{~h}$ at $37^{\circ} \mathrm{C}$ with $5 \% \mathrm{CO}_{2}$. Cotton swabs were used to remove the non-invasive cells on the upper chambers. The migrated cells on the lower membrane surface were fixed in $100 \%$ methanol at room temperature for $15 \mathrm{~min}$ and washed in PBS three times. Then, the migrated cells were stained using Hoechest 33342 at room temperature for $30 \mathrm{~min}$ and imaged using a light microscope (magnification, x100).

Cell invasion. Transwells ( $8.0 \mu \mathrm{m}$; BD Biosciences) were coated with diluted Matrigel (BD Biosciences) in PBS and dried at room temperature for $1 \mathrm{~h}$. The remaining steps followed the aforementioned protocol for the migration assays.

Reverse transcription-quantitative PCR (RT-qPCR). Total RNA was isolated from NCM460, SW620, HCT116, SW480 and HT29, SW480 cell-si-NC and SW480 cell-si-circRNA_101951 cells using TRIzol ${ }^{\circledR}$ reagent (Invitrogen; Thermo Fisher Scientific, Inc.) following the manufacturer's protocols. The mRNA expression levels of kinesin II family member 3A (KIF3A), E-cadherin, N-cadherin, vimentin and snail were examined using RT-qPCR. cDNA was synthesized using the qPCR RT kit (Toyobo Life Science) according to the manufacturers protocols. The temperature protocol for reverse transcription was as follows: $37^{\circ} \mathrm{C}$ for $15 \mathrm{~min}$ and $98^{\circ} \mathrm{C}$ for 5 min. The SYBR Green kit (Qiagen AB) was used for quantitative PCR. GAPDH was used for the internal control. The ABI Prism 7500 Sequence Detector (Applied Biosystems; Thermo Fisher Scientific, Inc.) was used for the PCR reactions. The thermocycling conditions were as follows: Denaturation at $95^{\circ} \mathrm{C}$ for $30 \mathrm{sec}$; followed by 40 cycles of annealing at $58^{\circ} \mathrm{C}$ for $30 \mathrm{sec}$ and extension at $72^{\circ} \mathrm{C}$ for $30 \mathrm{sec}$. The mRNA expression level was detected using the relative quantitative gene expression method (22). The primer sequences used are listed in Table I. 
Table I. Primer sequences.

\begin{tabular}{ll}
\hline Names & \multicolumn{1}{c}{ Sequence } \\
\hline circRNA_101951 & F, 5'-TTAGTGACAAGGATCCAGGCGG-3' and R, 5'-TCCTCAGAAGCAGCCAGTACATCTC-3' \\
KIF3A & F, 5'-ACGCTGACGACATGGATAGAATCATG-3' and R, 5'-CAGTTTGGAGTTCCGATACGGCAC-3' \\
E-cadherin & F, 5'-CGAGAGCTACACGTTCACGG-3' and R, 5'-GGGTGTCGAGGGAAAAATAGG-3' \\
N-cadherin & F, 5'-AGCCAACCTTAACTGAGGAGT-3' and R, 5'-GGCAAGTTGATTGGAGGGATG-3' \\
Vimentin & F, 5'-TGGCACGTCTTGACCTTGAA-3' and R, 5'-GGTCATCGTGATGCTGAGAA-3' \\
Snail & F, 5'-CCTTCTCTAGGCCCTGGCT-3' and R, 5'-AGGTTGGAGCGGTCAGC-3' \\
GAPDH & F, 5'-GAAGGTGAAGGTCGGAGTC-3' and R, 5'-GAAGATGGTGATGGGATTTC-3'
\end{tabular}

circRNA, circular RNA; KIF3A, kinesin II family member 3A; F, forward; R, reverse.

Western blot analysis. Total protein was extracted from treated cells using IP lysis butter (cat. no. P0013J; Beyotime Institute of Biotechnology). Subsequently, protein concentration was determined by performing a bicinchoninic acid assay assay (cat. no. A53225; Thermo Fisher Scientific, Inc.). Protein $(25 \mu \mathrm{g})$ samples were subjected to $8-12 \%$ SDS-PAGE and electro-transferred to PVDF membranes. The membranes were then blocked with $5 \%$ skimmed milk at room temperature for $2 \mathrm{~h}$ and incubated overnight at $4^{\circ} \mathrm{C}$ with primary antibodies against KIF3A (cat. no. sc-55598; 1:1,000), E-cadherin (cat. no. sc-8426; 1:1,000), N-cadherin (cat. no sc-8424; 1:1,000), vimentin (cat. no. sc-6260; 1:1,000), snail (cat. no. sc-393172; 1:1,000) and GAPDH (cat. no. sc-47724; 1:1,000). All primary antibodies were purchased from Santa Cruz Biotechnology, Inc. The membranes were subsequently incubated at room temperature for $2 \mathrm{~h}$ with horseradish peroxidase-conjugated anti-rabbit IgG secondary antibodies against anti-rabbit (cat. no. 7074; $1: 2,000$ ) and anti-mouse (cat. no. 7076; 1:2,000). All secondary antibodies were purchased from Cell Signaling Technology, Inc. Detection was performed using an ECL western blotting kit (GE Healthcare) with the ChemicDoc XRS system (Bio-Rad Laboratories, Inc.).

Statistical analysis. Statistical analysis was performed using SPSS (version 15.0; SPSS, Inc.) and GraphPad Prism 6 (GraphPad Software, Inc.). Student's t-test or one-way ANOVAs followed by a post-hoc Tukey test was performed for calculation of statistical differences. $\mathrm{P}<0.05$ was considered to indicate a statistically significant difference.

\section{Results}

CircRNA_101951 is upregulated in both CRC clinical specimens and cell lines. circRNAs were initially evaluated in CRC by retrieving the microarray data from the GEO dataset (GSE126094) and normalized microarray data was analyzed using GEO2R after applying a $\log 2$ transformation to the data. The microarray data demonstrated that the expression levels of circRNA_101951 were increased in CRC tissues compared with normal tissues (Fig. 1A). Furthermore, 62 pairs of CRC tissues and normal tissues were collected to detect circRNA_101951 expression levels. The results revealed that circRNA_101951 expression levels were significantly upregulated in CRC tissues compared with normal tissues $(\mathrm{P}<0.001$; Fig. 1B). Kaplan-Meier survival analysis indicated that higher expression levels of circRNA_101951 were associated with a lower survival rate in patients with CRC (Fig. 1C). The RT-qPCR assays demonstrated that circRNA_101951 was significantly upregulated in CRC cell lines compared with NCM460 cells $(\mathrm{P}<0.01$ or $\mathrm{P}<0.001$; Fig. 1D). SW480 cells were selected for further analysis as they exhibited the highest expression of circRNA_101951.

CircRNA_101951 exerts a tumor-promotive function in CRC. To assess the effect of circRNA_101951 on CRC cells, SW480 cells were transfected with si-NC or si-circRNA_101951. The RT-qPCR assays demonstrated that circRNA_101951 was significantly decreased in SW480 cell-si-circRNA_101951 compared with SW480 cell-si-NC ( $\mathrm{P}<0.001$; Fig. 2A). The MTT assays revealed that cell proliferation was significantly inhibited in SW480 cell-si-circRNA_101951 compared with SW480 cell-si-NC $(\mathrm{P}<0.001$; Fig. 2B). Furthermore, the clone formation ability of the cells was significantly reduced in SW480 cell-si-circRNA_101951 (P<0.001; Fig. 2C). Subsequently, the flow cytometry analysis was performed to further detect the effect of circRNA_101951 on CRC cell apoptosis and the cell cycle progression. The results indicated that the rate of apoptosis was remarkably increased when SW480 cells were transfected with si-circRNA_101951 ( $\mathrm{P}<0.001$; Fig. 2D). The proportion of G1 cells was markedly increased following circ_101951 knockdown and the proportion of cells in the G2 phase was markedly decreased after circ_101951 knockdown, indicating G1 arrest $(\mathrm{P}<0.001$; Fig. 2E). Therefore, circRNA_101951 may exert a tumor-promotive function in CRC.

Downregulation of circRNA_101951 suppresses SW480 cell migration and invasion in vitro. In the present study, the overexpression of circRNA_101951 was indicated to be associated with metastasis, which suggested that circRNA may promote CRC migration and invasion (Table II). To further verify whether circRNA_101951 promoted CRC migration and invasion, cell migration and invasion assays were performed to examine the migration and invasion in CRC cells transfected with si-NC or si-circRNA_101951. The results showed that downregulation of circRNA_101951 significantly inhibited the migratory $(\mathrm{P}<0.001 ;$ Fig. $3 \mathrm{~A})$ and invasive $(\mathrm{P}<0.001$; 
A
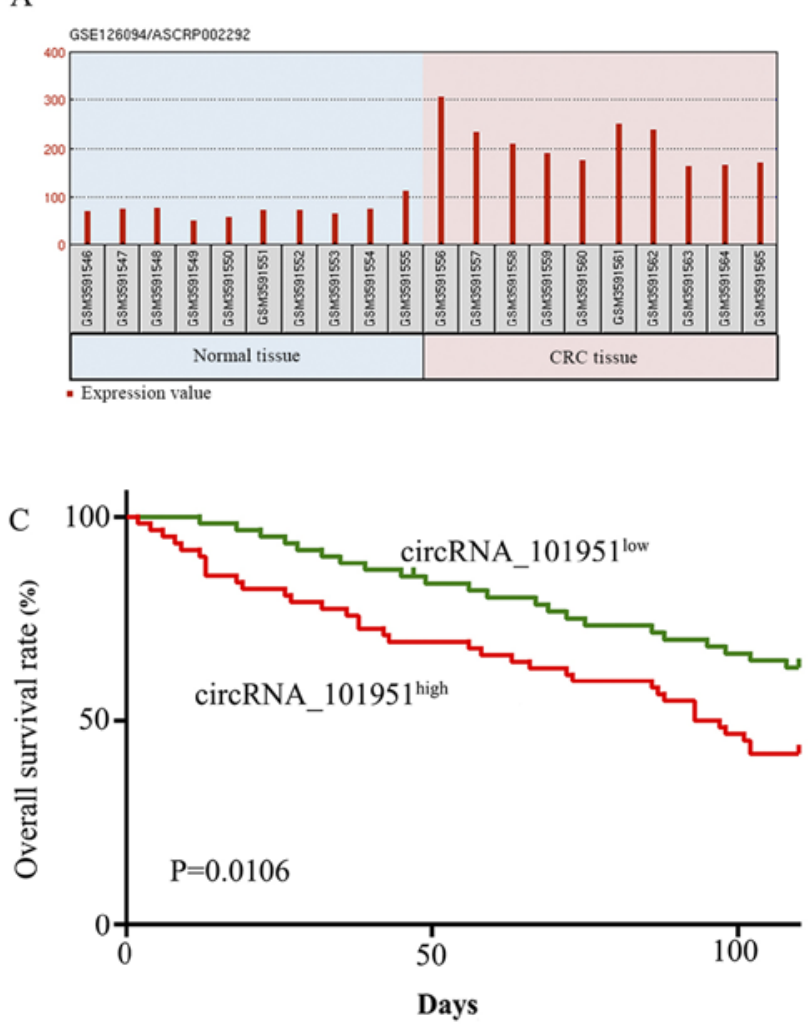

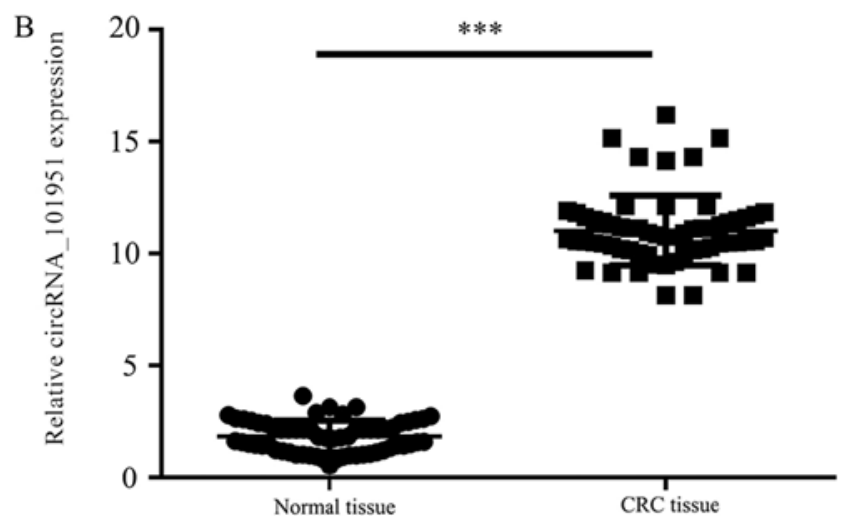

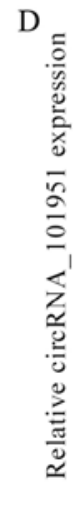

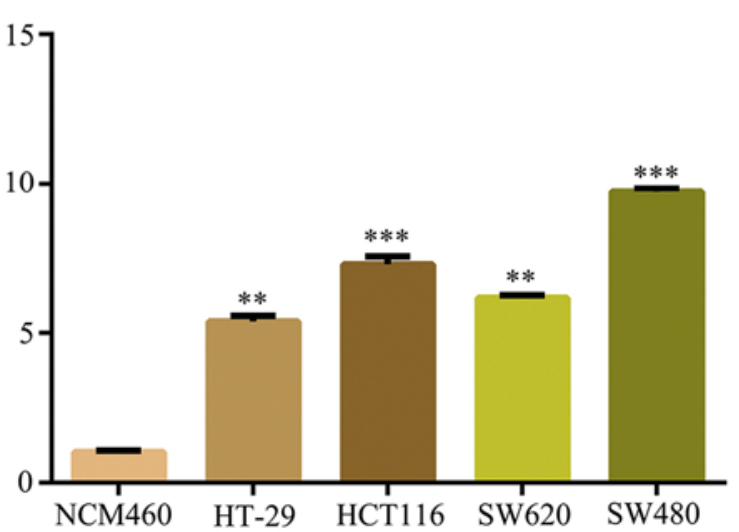

Figure 1. circRNA_101951 is upregulated in both CRC clinical specimens and cell lines. (A) The GEO dataset (GSE126094) showed that circRNA_101951 was significantly upregulated in tumor samples compared with normal samples. (B) circRNA_101951 expression levels were examined using RT-qPCR in CRC samples and normal samples. (C) Kaplan-Meier survival analysis. (D) circRNA_101951 were examined using RT-qPCR in the CRC cell lines HT-29, HCT116, SW620 and SW480 and the normal colonic epithelial cells NCM460. The data shown represents the mean \pm standard deviation $(\mathrm{n}=3) .{ }^{* *} \mathrm{P}<0.01,{ }^{* * *} \mathrm{P}<0.001$ vs. the NCM460 cells. circRNA, circular RNA; CRC, colorectal cancer; KIF3a, kinesin II family member 3A; RT-qPCR, reverse transcription-quantitative PCR.

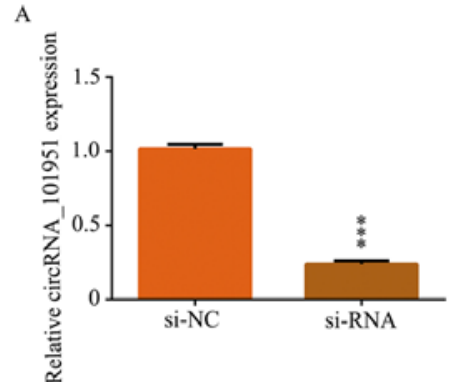

D

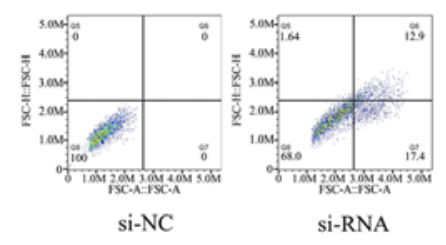

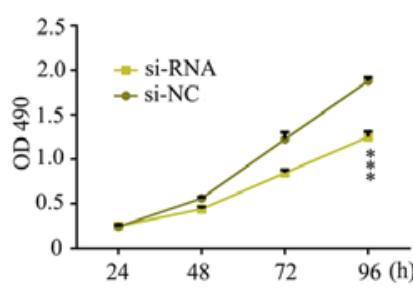

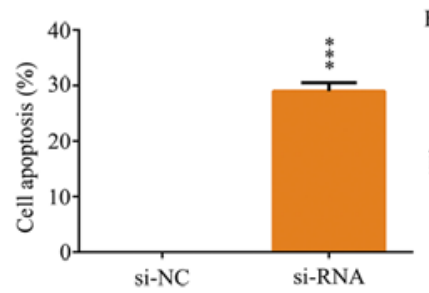

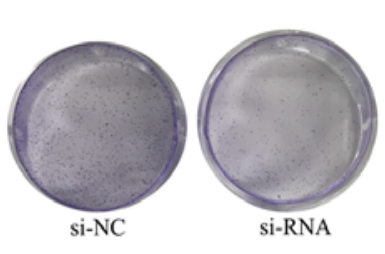
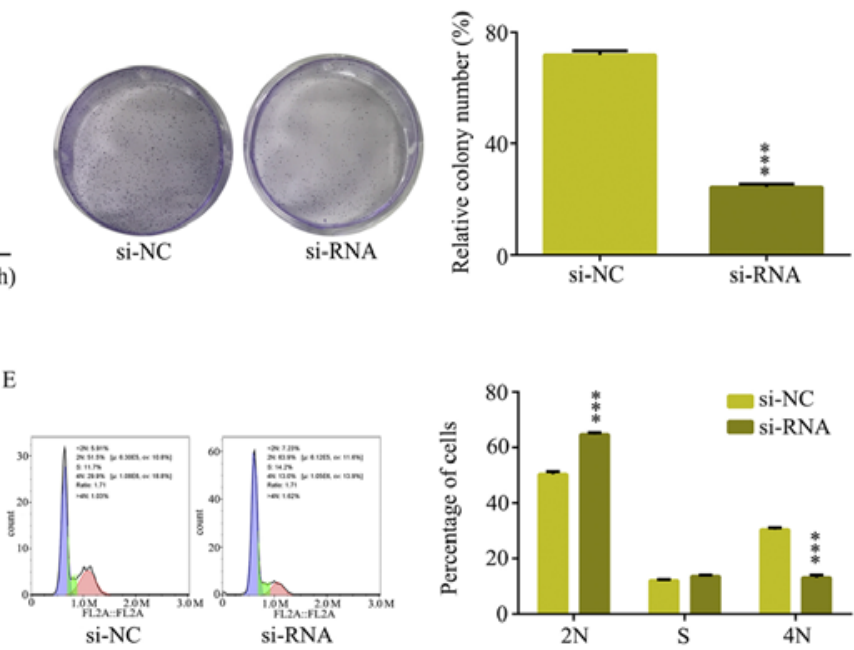

Figure 2. circRNA_101951 exerted a tumor-promotive function in colorectal cancer. si-RNA targeting circRNA_101951 was transfected in SW480 cells. (A) circRNA_101951 expression levels were examined after transfection in SW480 cells using reverse transcription-quantitative PCR. (B) MTT assays were performed to examine the cell viability of SW480 cells after transfections. (C) Colony formation assays were performed to examine the clone ability of SW480 cells after transfection. (D) Flow cytometric analysis were performed to examine cell apoptosis of SW480 cells after transfection. (E) Flow cytometric analyses were performed to examine cell cycle progression of SW480 cells after transfection. The data shown represent the mean \pm standard deviation $\left(\mathrm{n}=3\right.$ ). ${ }^{* * *} \mathrm{P}<0.001$ vs. the si-NC group. circRNA, circular RNA; OD, optical density; si-NC, negative control small interfering RNA; si-RNA, small interfering RNA.

Fig. 3B) abilities of SW480. Therefore, circRNA_101951 exerted a promotive function on the migration and invasion of CRC cells.
CircRNA_101951 modulates EMT of CRC cells. The EMT is a critical cellular progress in tumor migration and invasion. Therefore, to detect the effect of circRNA_101951 
Table II. Relationship between clinical features and circRNA_101951 expression in patients with colorectal cancer.

\begin{tabular}{|c|c|c|c|c|}
\hline \multirow[b]{2}{*}{ Number of variables } & \multirow[b]{2}{*}{ Cases } & \multicolumn{2}{|c|}{ circRNA_101951 expression } & \multirow[b]{2}{*}{ P-value } \\
\hline & & Low & High & \\
\hline Age (years) & & & & 0.349 \\
\hline$<55$ & 33 & $20(9.94 \pm 1.56)$ & $13(12.09 \pm 0.39)$ & \\
\hline$\geq 55$ & 29 & $11(9.48 \pm 0.97)$ & $18(12.26 \pm 0.61)$ & \\
\hline Sex & & & & 1.739 \\
\hline Male & 32 & $17(9.57 \pm 0.87)$ & $15(12.11 \pm 0.55)$ & \\
\hline Female & 30 & $14(9.33 \pm 1.12)$ & $16(11.93 \pm 0.73)$ & \\
\hline TNM stage & & & & $<0.01$ \\
\hline I-II & 25 & $23(9.75 \pm 0.58)$ & $2(10.79 \pm 0.83)$ & \\
\hline III-IV & 37 & $8(10.23 \pm 0.75)$ & $29(12.56 \pm 1.25)$ & \\
\hline Tumor size & & & & $<0.01$ \\
\hline$<5 \mathrm{~cm}$ & 24 & $20(9.56 \pm 0.29)$ & $4(10.56 \pm 0.57)$ & \\
\hline$\geq 5 \mathrm{~cm}$ & 38 & $11(9.27 \pm 1,12)$ & $27(12.63 \pm 0.95)$ & \\
\hline Metastasis & & & & $<0.01$ \\
\hline No & 30 & $24(9.36 \pm 0.82)$ & $6(10.25 \pm 0.81)$ & \\
\hline Yes & 32 & $7(9.73 \pm 1.34)$ & $25(13.051 \pm 28)$ & \\
\hline
\end{tabular}

circRNA, circular RNA; TNM, tumor node metastasis.

A

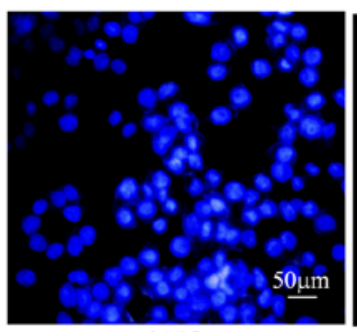

si-NC

B

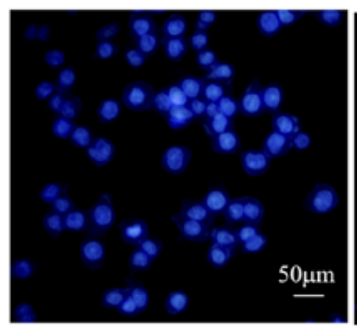

si-NC

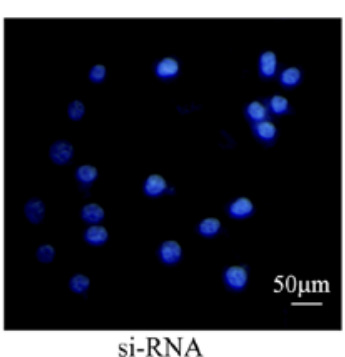

si-RNA

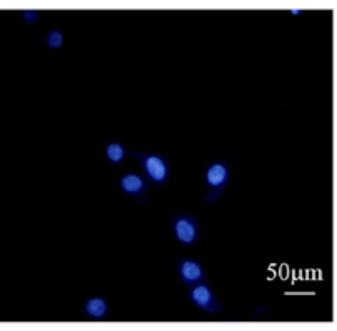

si-RNA
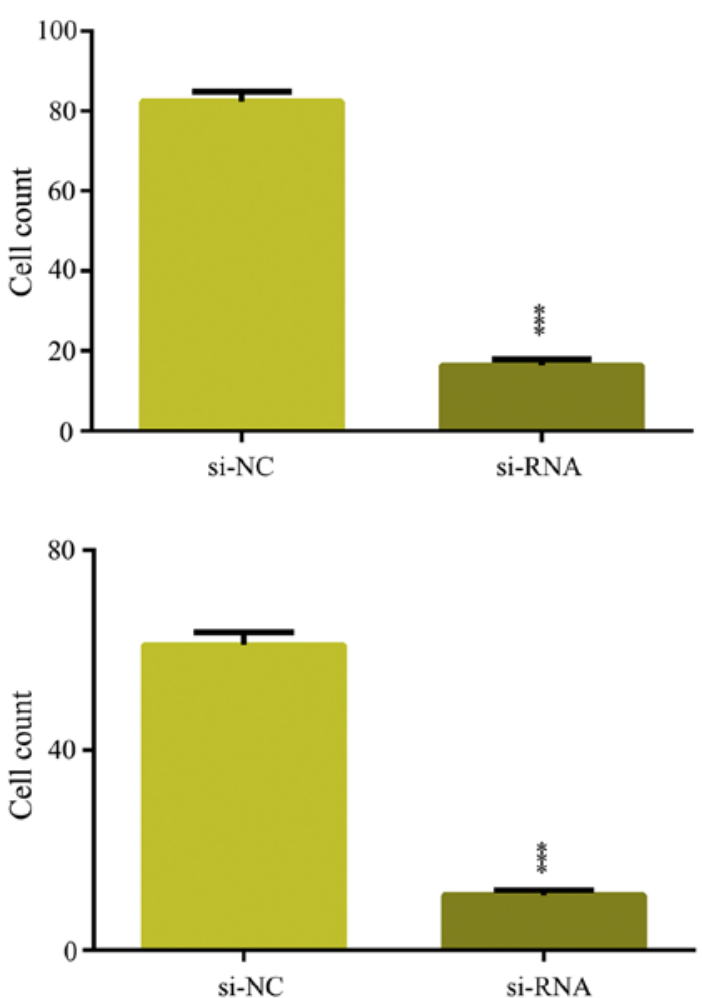

Figure 3. Downregulation of circular RNA_101951 suppressed SW480 cell migration and invasion in vitro. si-RNA targeting circRNA_101951 was transfected into SW480 cells. (A) Transwell assays were performed to examine the cell migratory capacity of SW480 cells following transfection. (B) Transwell assays were performed to examine the cell invasive capacity of SW480 cells after transfection. The data shown represent the mean \pm standard deviation (n=3). ${ }^{* * *} \mathrm{P}<0.001$. si-NC, negative control small interfering RNA; si-RNA, small interfering RNA.

suppression on EMT pathways, RT-qPCR and western blot assays were performed. It was demonstrated that
circRNA_101951 inhibitor decreased the mRNA and protein expression levels of KIF3A as well as mesenchymal 

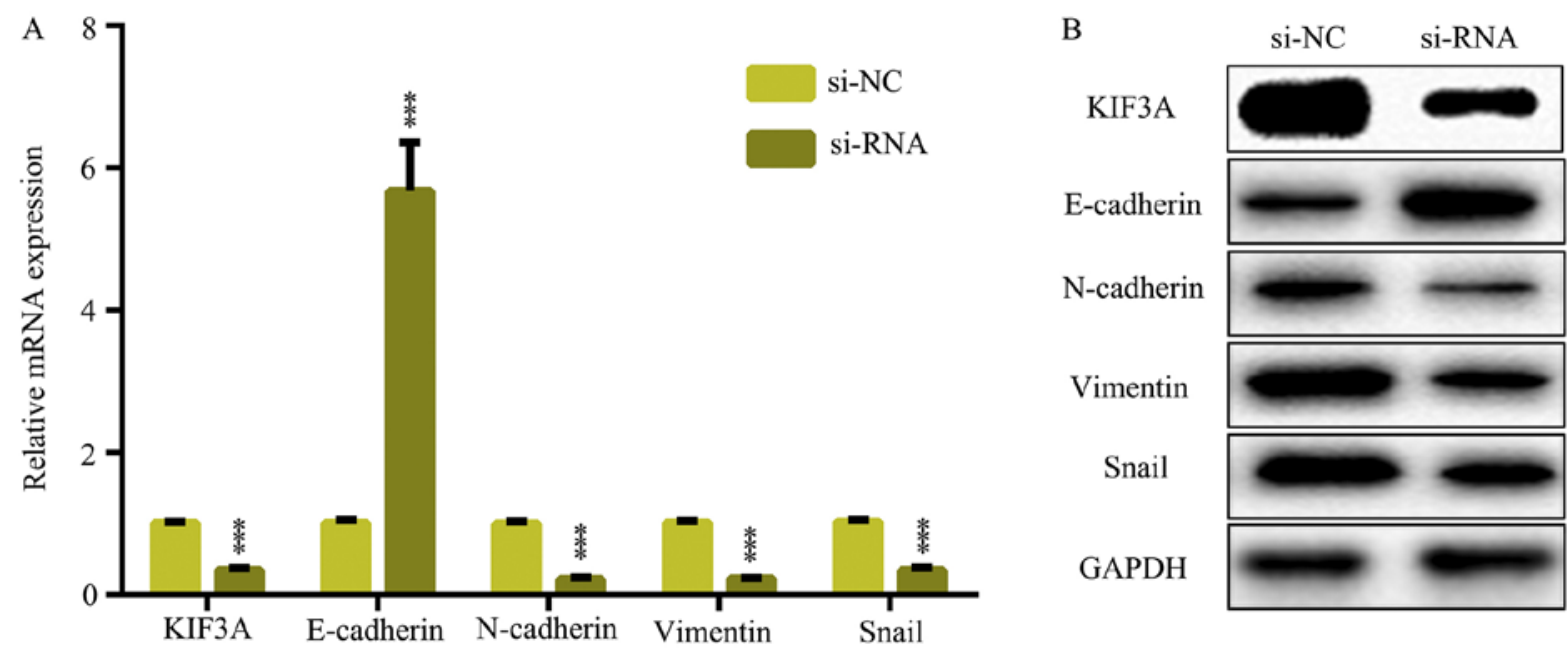

Figure 4. circRNA_101951 modulates EMT of CRC cells. (A) Reverse transcription-quantitative PCR was used to examine the mRNA expression levels of KIF3A, E-cadherin, vimentin, N-cadherin and snail in SW480 cells transfected with si-circRNA_101951 or si-NC. (B) Western blotting was used to examine the protein expression levels of KIF3A, E-cadherin, vimentin, N-cadherin and snail in SW480 cells transfected with si-circRNA_101951 or si-NC. The data shown represent the mean \pm standard deviation $(n=3) .{ }^{* * *} \mathrm{P}<0.001$. circRNA, circular RNA; KIF3a, kinesin II family member 3A; si-NC, negative control small interfering RNA; si-RNA, small interfering RNA.

marker genes including $\mathrm{N}$-cadherin, vimentin and snail and increased the mRNA and protein expression levels of the epithelial marker gene, E-cadherin ( $\mathrm{P}<0.05$; Fig. 4A and B). Thus, circRNA_101951 promoted the migration and invasion of CRC by activating the KIF3A-mediated EMT signaling pathway.

\section{Discussion}

CRC development is a complex, dynamic and biological process, and is associated with multiple genes. In addition, a variety of circRNAs have been reported as either oncogenic or tumor suppressive factors. For example, circRNA hsa_circ_000984 promotes colon cancer growth and metastasis by sponging miR-106b (23). circRNA hsa_circ_0008039 promotes breast cancer cell proliferation and migration by regulating the miR-432-5p/E2F3 axis (24). Hsa_circ_0079530 promotes cell proliferation and invasion in non-small cell lung cancer (25). Therefore, it is important to study the molecular mechanisms of circRNAs in CRC.

Recently, it has been demonstrated that circRNAs are useful as molecular biomarkers. For example, hsa_circ_0013958 is a potential novel biomarker for lung adenocarcinoma (15), circPVT1 is a potential novel biomarker in osteosarcoma (26) and hsa_circ_0005986 acts as a novel biomarker for hepatocellular carcinoma (27). In addition, circRNAs also act as a sponge to inhibit the functions of miRNA. For example, hsa_circ_000984 promotes colon cancer growth and metastasis by sponging miR-106b (23). Increased circRNA hsa_circ_0012673 acts as a sponge of miR-22 to promote lung adenocarcinoma proliferation (20). Hsa_circRNA_0006528 acts as a competing endogenous RNA and promotes human breast cancer progression by sponging miR-7-5p and activating the MAPK/ERK signaling pathway (28). In the present study, a novel circRNA, circRNA_101951, was identified and its function was examined. The present study is, to the best of our knowledge, the first report showing that circRNA_101951 promoted cell proliferation, migration and invasion as well as inhibiting cell apoptosis in CRC. In addition, RT-qPCR and western blot assays revealed that circRNA_101951 promoted migration and invasion of CRC by activating the KIF3A-mediated EMT signaling pathway. Therefore, circRNA_101951 exerts a tumor-promotive function and is a promising biomarker for CRC.

In conclusion, circRNA_101951 is upregulated and associated with the migration and invasion ability of CRC cells. In addition, circRNA_101951 may promote CRC cell migration and invasion by activating the KIF3A-mediated EMT signaling pathway. Therefore, the present data revealed that circRNA_101951 may act as a potential biomarker for patients with CRC and provide a novel insight in to a potential therapeutic strategy for CRC.

\section{Acknowledgements}

Not applicable.

\section{Funding}

No funding received.

\section{Availability of data and materials}

The datasets used and/or analyzed in the current study are available from the corresponding author on reasonable request.

\section{Authors' contributions}

FLP conceived and designed the current study and drafted the manuscript. YFL performed the experiments and analyzed the data. MZC also performed the experiments. All authors read and approved the final manuscript. 


\section{Ethics and approval and consent to participate}

Experiments involving patient tissues were approved by The Ethics Committee of Linyi Central Hospital for Clinical Ethical Review. Written consent was provided by all enrolled patients.

\section{Patient consent for publication}

Not applicable.

\section{Competing interests}

The authors declare that they have no competing interests.

\section{References}

1. Siegel RL, Miller KD and Jemal A: Cancer statistics, 2018. CA Cancer J Clin 68: 7-30, 2018.

2. Bopanna S, Ananthakrishnan AN, Kedia S, Yajnik V and Ahuja V: Risk of colorectal cancer in Asian patients with ulcerative colitis: A systematic review and meta-analysis. Lancet Gastroenterol Hepatol 2: 269-276, 2017.

3. Longo WE, Virgo KS, Johnson FE, Oprian CA, Vernava AM, Wade TP, Phelan MA, Henderson WG, Daley J and Khuri SF: Risk factors for morbidity and mortality after colectomy for colon cancer. Dis Colon Rectum 43: 83-91, 2000.

4. Kube R, Mroczkowski P, Granowski D, Benedix F, Sahm M, Schmidt U, Gastinger I and Lippert H; Study group Qualitätssicherung Kolon/Rektum-Karzinome (Primärtumor) (Quality assurance in primary colorectal carcinoma) Anastomotic leakage after colon cancer surgery: A predictor of significant morbidity and hospital mortality, and diminished tumour-free survival. Eur J Surg Oncol 36: 120-124, 2010.

5. Qu S, Yang X, Li X, Wang J, Gao Y, Shang R, Sun W, Dou K and Li H: Circular RNA: A new star of noncoding RNAs. Cancer Lett 365: 141-148, 2015

6. Zhang HD, Jiang LH, Hou JC, Zhong SL, Zhou SY, Zhu LP, Li J, Wang DD, Sun DW, Ji ZL and Tang JH: Circular RNA hsa circ 0052112 promotes cell migration and invasion by acting as sponge for miR-125a-5p in breast cancer. Biomed Pharmacother 107: 1342-1353, 2018.

7. Zhang M, Xia B, Xu Y, Zhang Y, Xu J and Lou G: Circular RNA (hsa_circ_0051240) promotes cell proliferation, migration and invasion in ovarian cancer through miR-637/KLK4 axis. Artif Cells Nanomed Biotechnol 47: 1224-1233, 2019.

8. Zhang XL, Xu LL and Wang F: Hsa_circ_0020397 regulates colorectal cancer cell viability, apoptosis and invasion by promoting the expression of the miR-138 targets TERT and PD-L1. Cell Biol Int 41: 1056-1064, 2017.

9. Zhang Y, Liang W, Zhang P, Chen J, Qian H, Zhang X and Xu W: Circular RNAs: Emerging cancer biomarkers and targets. J Exp Clin Cancer Res 36: 152, 2017.

10. Zhang Z, Xie Q, He D, Ling Y, Li Y, Li J and Zhang H: Circular RNA: New star, new hope in cancer. BMC Cancer 18: 834, 2018.

11. Cortes-Lopez M and Miura P: Emerging functions of circular RNAs. Yale J Biol Med 89: 527-537, 2016.

12. Han YN, Xia SQ, Zhang YY, Zheng JH and Li W: Circular RNAs: A novel type of biomarker and genetic tools in cancer. Oncotarget 8: 64551-64563, 2017.

13. Sun H, Tang W, Rong D, Jin H, Fu K, Zhang W, Liu Z, Cao H and Cao X: Hsa_circ_0000520, a potential new circular RNA biomarker, is involved in gastric carcinoma. Cancer Biomark 21: 299-306, 2018
14. Shang X, Li G, Liu H, Li T, Liu J, Zhao Q and Wang C: Comprehensive circular RNA profiling reveals that hsa circ 0005075, a new circular RNA biomarker, is involved in hepatocellular crcinoma development. Medicine (Baltimore) 95: e3811, 2016.

15. Zhu X, Wang X, Wei S, Chen Y, Chen Y, Fan X, Han S and Wu G: hsa_circ_0013958: A circular RNA and potential novel biomarker for lung adenocarcinoma. FEBS J 284: 2170-2182, 2017.

16. Liu T, Liu S, Xu Y, Shu R, Wang F, Chen C, Zeng Y and Luo H: Circular RNA-ZFR inhibited cell proliferation and promoted apoptosis in gastric cancer by sponging miR-130a/miR-107 and Modulating PTEN. Cancer Res Treat 50: 1396-417, 2018.

17. Rong D, Lu C, Zhang B, Fu K, Zhao S, Tang W and Cao H: CircPSMC3 suppresses the proliferation and metastasis of gastric cancer by acting as a competitive endogenous RNA through sponging miR-296-5p. Mol Cancer 18: 25, 2019.

18. Bai N, Peng E, Qiu X, Lyu N, Zhang Z, Tao Y, Li X and Wang Z circFBLIM1 act as a ceRNA to promote hepatocellular cancer progression by sponging miR-346. J Exp Clin Cancer Res 37: $172,2018$.

19. Greene FL, Stewart AK and Norton HJ: A new TNM staging strategy for node-positive (stage III) colon cancer: An analysis of 50,042 patients. Ann Surg 236: 416-421, 2002.

20. Wang X, Zhu X, Zhang H, Wei S, Chen Y, Chen Y, Wang F, Fan X, Han S and Wu G: Increased circular RNA hsa_circ_0012673 acts as a sponge of miR-22 to promote lung adenocarcinoma proliferation. Biochem Biophys Res Commun 496: 1069-1075, 2018.

21. Zhou LH, Yang YC, Zhang RY, Wang P, Pang MH and Liang LQ: CircRNA_0023642 promotes migration and invasion of gastric cancer cells by regulating EMT. Eur Rev Med Pharmacol Sci 22: 2297-2303, 2018

22. Livak KJ and Schmittgen TD: Analysis of relative gene expression data using real-time quantitative PCR and the 2(-Delta Delta C(T)) method. Methods 25: 402-408, 2001.

23. Xu XW, Zheng BA, Hu ZM, Qian ZY, Huang CJ, Liu XQ and Wu WD: Circular RNA hsa_circ_000984 promotes colon cancer growth and metastasis by sponging miR-106b. Oncotarget 8: 91674-91683, 2017.

24. Liu Y, Lu C, Zhou Y, Zhang Z and Sun L: Circular RNA hsa_circ_0008039 promotes breast cancer cell proliferation and migration by regulating miR-432-5p/E2F3 axis. Biochem Biophys Res Commun 502: 358-363, 2018.

25. Li J, Wang J, Chen Z, Chen Y and Jin M: Hsa_circ 0079530 promotes cell proliferation and invasion in non-small cell lung cancer. Gene 665: 1-5, 2018

26. Kun-Peng Z, Xiao-Long $M$ and Chun-Lin Z: Overexpressed circPVT1, a potential new circular RNA biomarker, contributes to doxorubicin and cisplatin resistance of osteosarcoma cells by regulating ABCB1. Int J Biol Sci 14: 321-330, 2018.

27. Fu L, Chen Q, Yao T, Li T, Ying S, Hu Y and Guo J: Hsa circ_0005986 inhibits carcinogenesis by acting as a miR-129-5p sponge and is used as a novel biomarker for hepatocellular carcinoma. Oncotarget 8: 43878-43888, 2017.

28. Gao D, Qi X, Zhang X, Fang K, Guo Z and Li L: hsa circRNA_0006528 as a competing endogenous RNA promotes human breast cancer progression by sponging miR-7-5p and activating the MAPK/ERK signaling pathway. Mol Carcinog 58: $554-564,2019$

(i) $\ominus$ This work is licensed under a Creative Commons Attribution-NonCommercial-NoDerivatives 4.0 International (CC BY-NC-ND 4.0) License. 